\title{
Exploration on Research Trends of Curriculum and Teaching Methodology at New Era
}

\author{
Yongjian Huang ${ }^{1}$, Xiaobo Zhang ${ }^{2}$ \\ ${ }^{1}$ Polytechnic Institute, Jiangxi Science and Technology Normal University, Nanchang Jiangxi, \\ 330108, China \\ ${ }^{2}$ Nanchang Academy of Social Sciences, Nanchang Jiangxi, 330038, China
}

Keywords: New ear, Curriculum and teaching methodology, Research trends, Practicality

\begin{abstract}
Curriculum teaching methodology has been developed for a long time, after the continuous improvement and development, in the last century it has been rapid development, but from another point of view, there are still a series of problems to be improved, which is particularly prominent in the curriculum and teaching On the theoretical basis of the rich, but the lack of practice. This deficiency tendency proposed curriculum and teaching methodology of integration has been more and more concern and attention of scholars, which has been providing for curriculum and teaching methodology development a broader space for development, marks the curriculum and teaching methodology into a new stage of development, and provides a way for innovative practice, further reveals the teacher behavior. Thus, the paper mainly on research trends in the New Curriculum and Teaching methodology analysis, combined with the actual situation, which objectively describes deficiencies exist, a reasonable countermeasures.
\end{abstract}

\section{Introduction}

Curriculum and Teaching is one of the indispensable disciplines of education branches, primarily related to research and practice for school education curriculum and teaching, through the continuous improvement and development, with psychology, sociology, cultural studies exists between anthropology and other disciplines as well as a close relationship, and with the progress and development of society, gradually toward theoretical multidisciplinary research directions higher levels of development. In addition, the pace of new curriculum standards to promote education reform, curriculum and teaching theory for the study of the rapid rise of education discipline curriculum and teaching theory gained new historical opportunity, but the corresponding also faces enormous challenges. Seen in this light, strengthen the curriculum and teaching theory research is very necessary to help provide a reference for future theoretical research.

\section{Inadequate practice character of curriculum and teaching methodology}

Curriculum theory and teaching born in the practice, through the continuous improvement and innovation to achieve a more substantial effect until the nineties of the last century, the introduction of curriculum and teaching in China 's Theory, the actual level of education, through continuous innovation and improvement, has become the rapid development of postgraduate training discipline, curriculum and teaching theory, to a certain extent, enrich the teaching discipline, to create a more rich professional knowledge, but from another perspective, the rapid development of curriculum and teaching methodology. It exposed an important issue, namely the practice of character deficiencies, theory and practice from theory to practice not effectively play a guiding role in [1].

\section{Theory is broken away from practice}

On the educational development process of China's point of view, from the theory with practice has become a universal phenomenon, has become recognized as one of the areas of education to be solving difficult problems. Subject crisis existing in the field of education, curriculum and teaching theory in somewhat the same performance, theory and practice of contact is not close, deviate from 
phenomenon is particularly prominent. Our curriculum and teaching methodology through constant innovation and improvement, more solid theoretical basis, from an abstract point of view to talk about humanism and constructivism, to a certain extent for curriculum and teaching methodology enrich some big words. Since the origin of these big words earlier, through continuous research and improvement has a relatively rich theoretical basis and can advance with the times, absorbing elements of modern society, and improve curriculum and teaching methodology itself, thereby forming a new theory. However, the current situation from the practical application of these big words just stay on the surface level, in practice, has not been fully reflected detachment theory with practice still exists [2].

Curriculum and Teaching Theory from theory with practice for the development poses a serious crisis, there have been numerous derivative factions in the historical process, there are differences between different factions theoretical characteristics, but in general point of view greatly enriched the curriculum and teaching On a theoretical basis, but there are also subjects of digestion problems when evaluating the results of such research, curriculum and teaching theory is not difficult to find problems in other disciplines by erosion, against such phenomena, there are few scholars have felt the curriculum and teaching On the soon-to-face threatening and trying to take reasonable measures to solve the problem to solve.

One of the main areas of curriculum and teaching theory is the study of the behavior of teachers, to a certain extent, it reveals the specific performance combination of theory and practice, current research on self-understanding and teacher behavior observed behavior less, stay in the shallow level It has not been able to form a strong theoretical basis, so that there has been an awkward situation in which theoretical research curriculum and teaching theory of wealth, to expand teacher training and education deeper, but in primary and secondary education in practice rarely mentioned, eventually leading to curriculum and teaching methodology practice conduct is not high [3].

\section{Theory lacks of guidance for practice}

On the current situation on the curriculum and teaching development, the theory for practice is not strong more and more problems, many theoretical concepts confusion, lack of practical significance of such concepts, lack of education activities guiding role in the practice range Applications are also becoming less and less effective inside. New Curriculum education reform advocates such as the quality of education in order to stimulate students 'interest mainly interested in becoming key issues that most concern to school teachers, some teachers in the analysis of the students' interest primarily with their own teaching experience, interest in the practice of the specific content and how cultivate interest in learning other aspects are poorly understood [4]. When this problem, cannot help people on the research and education expertise to produce a strong challenge, to acquire more expertise in theory have any practical effect. In curriculum and teaching methodology course of development, curriculum theory and teaching theory into each faction, formed a relatively rich theoretical basis, but on the relationship between theory and practice, the curriculum and teaching Theoretical knowledge of teacher behavior not play positive effect, but the emergence of a more theoretical knowledge rich, the more abstract, for teachers, teaching this theory in practice are more and more weakened. Overall, this phenomenon, with the main structures and systems to improve the knowledge has more deep-rooted ties to be further tapped.

Curriculum and Teaching methodology researchers because of their lack of practice awareness, lack of practical experience teaching primary and secondary schools, so it is difficult to be more abundant and vivid teaching content as a new theory, based on a theoretical level, although the theory proposed content more autonomy, but from the perspective of school teachers, this lack of integration of theory with practice, theory and practice of contact is not close, some school teachers for outstanding teaching experience severely curbed, not widely promoted and applications provide a reference for curriculum and teaching methodology of improvement and innovation [5]. In addition, curriculum and teaching methodology practices, not only in terms of the conduct of insufficient knowledge of the structure, but also in the construction of the way the problem of irrational, curriculum and teaching methodology focus more on discovery and analysis of the problem, in this 
paradigm, the more timely was independent segmented, teachers had to teach students in teaching knowledge, develop good study its role in literacy are ignored, even in teachers' professional studies, barely mentioned teacher behavior, resulting in disjointed practice and theory, curriculum and teaching methodology Teachers' Behavior Study of missing practice also reduces the character, is not conducive to the New Curriculum Course System Reform.

\section{Research direction of curriculum and teaching methodology - teachers' behavior study}

Teacher behavior mainly refers to teachers in the teaching activities, practical activities under the guidance of the concept of curriculum and teaching methodology undertaken, teacher behavior is a theoretical construct, as a kind of culture, and from the perspective of teachers and curriculum culture coordinates development of teachers and between students, provide more professional analysis framework for teacher behavior. In essence, the help to break the traditional teaching philosophy, teaching methods, teaching content, to create a good educational environment, teachers act rationality depth exploration and cultural interpretation. For teachers conduct research, on the one hand it can be seen as the integration of research curriculum and teaching theory, on the other hand it is seen as a historical text study, but no matter what kind of research perspective, studies the behavior of teachers Education will be based on the use of knowledge as the theoretical basis for the development stage of all-round teacher behavior analysis. Seen in this light, teacher behavior not only covers the curriculum theory of property, but also includes the content of teaching theory, is an important method and means to integrate curriculum and teaching theory.

\section{Establishment of integration of curriculum theory and teaching methodology}

Research on teacher behavior is mainly followed in teaching theory and curriculum theory formed the basis of the construction of a new, multi-course curriculum text and discourse coexist, when the researchers combined different theoretical basis for analysis of teacher behavior, teacher behavior is a generalized nature The research that can highlight the psychological characteristics, but also to highlight the characteristics of education, and even the integration of many disciplines to use the content. This construction of way, for the theoretical development of educational discipline has a more profound impact, but also easily lead to disciplines Curriculum and Teaching methodology direction deviation, affecting their practice character. Overall, teacher behavior was able to become curriculum and teaching theory practice an important part character study, mainly by teacher behavior characteristics of the decision itself, primary and secondary teachers is to guide and organizer of classroom activities, but also the course and implementation of the main teaching and promote student development, teaching status characteristics of such activities, directly determine the basic characteristics of teacher behavior.

The relationship between teachers and students, the teacher mainly plays a leading role, in order to encourage students to get better education, to develop good learning ability, teachers need to give full play to their role in the development of teaching combined with the actual needs of the teaching program, have Targeted to promote the comprehensive development of the overall quality of students. Seen in this light, the overall quality of teachers is to train students of the main force, teacher behavior is the practical realization of the aims of education, teacher education reform as a direct participant, but also the integration of curriculum and teaching methodology Practical subject. As a new teacher behavior construct its study will help the professional development of teachers indicated the way forward to help teachers more profound reflection of their own lack of education exist, and constantly improve themselves in the role. Based on this, the effectiveness of the Institute teacher behavior made inevitable than the study of teachers' actions, the theoretical research value and practical value of organic character together, indicate a new direction.

\section{Promote integration of curriculum resources}

Research on teacher behavior, not only indicate the direction of teacher professional development, but also help to integrate curriculum resources, after the new curriculum standards of education reform, promote comprehensive development of students, teachers should give full play to its own 
guide and organizer, able to deal with professional quality teaching needs, integrating teaching resources for students to create a good learning atmosphere. With the development of science and technology, and resources became more varied, but the resources are not the same curriculum resources is the same concept, there are essential differences between the two, will be seen as a means of obtaining material resources or conditions in order to be translated into curriculum resources, integration of curriculum resources. Resources into curriculum resources is not a single condition of transformation, but to change the consciousness determines resources into curriculum resources depends on the concept of access to resources of the body. It is not difficult to see, the integration of curriculum resources for curriculum and teaching methodology development has a more prominent role in the transformation process resource is determined by the education body, so reasonable and effective determination of resources.

On the development of curriculum and teaching abroad longer history, through constant innovation and improvement, has made more impressive results, our country is the introduction of this theory in the 1990s, the concept of different courses, there are courses in resources obvious choice different. For example, children experience growing curriculum view, supported by the experiences of children growing up accumulated seen as curriculum resources to help students better absorb curriculum resources. Different theoretical perspectives will produce different evaluations of their cognitive teaching theory be regarded as the emphasis on students cognitive process, to a certain extent, compensate for the behavior of teaching theory in the presence of defects; the humanistic curriculum theory focus on talent The full potential and achieve self-worth. Therefore, the students' classroom learning content and significance of the creation of future development become indispensable curriculum resources.

In addition, the study of teachers' behavior can help improve and enrich the teaching theory, as an important research direction of development of teaching theory, the study of the behavior of teachers is seen as an independent research, which helps teaching basic theory, teaching the same Teaching Practice produce closer ties for teachers professional development direction. Foreign curriculum and teaching theory research Compared to the theory of this study later, after constant innovation and improvement, has made more impressive results in practice, but practical problems remain insufficient for this class of problems, strengthen the integration of theory with practice, in order to carry out practical work in full play to the guiding role of theory, lay the foundation for sustainable development of China's educational undertakings.

\section{Conclusions}

In summary, despite the introduction of curriculum and teaching methodology of late, but after the continuous improvement and innovation, has made more substantial achievements in theoretical research, but from the overall perspective, the theory with practice divorced from the phenomenon exists , the lack of practical character, this issue has become a priority to be the development of curriculum and teaching theory to solve one of the problems. The research on teacher behavior helps curriculum and teaching methodology really one and, to strengthen the practice character, lay the foundation for academic development, cultivate more excellent talents Master of Education.

\section{References}

[1] Dai Shuping. Independence and entanglement: China's curriculum and teaching methodology research from 1949-1977. Shanxi Normal University, 2014.

[2] Xu Jinjin. A Comparative Study Chinese and American music teacher education curriculum in cultural perspective. Hunan Normal University, 2014.

[3] Xin Yang. Construction of PE teaching theory and method at new era-Comment for Physical Education Curriculum and Teaching Methodology. Modern Education Science, 2015, 35(17): 71-72. 
[4] Yu Haijie. On primary mathematics curriculum and teaching methodology construction in teachers’ college. Journal of Chifeng College (natural science edition), 2011(02): 264-266.

[5] Li Jianglin. Thoughts on Teachers' College Curriculum and Teaching Theory Discipline and Degree Construction. Journal of Hubei Normal College (philosophy and social sciences ed), 2013(05): 125-128. 